\title{
FRICTIONAL CONTACT OF TWO SOLIDS WITH A PERIODICALLY GROOVED SURFACE IN THE PRESENCE OF AN IDEAL GAS IN INTERFACE GAPS
}

\author{
Nataliya Malanchuk, Bogdan Slobodyan, Rostyslav Martynyak \\ Pidstryhach Institute for Applied Problems of Mechanics and Mathematics, NASU, Lviv, Ukraine \\ e-mail: labmtd@iapmm.lviv.ua
}

\begin{abstract}
The frictional contact between two solids, one of which having a periodically grooved surface, under the action of normal and shear load is investigated. The interface between the contacting solids consists of a periodic array of gas-filled gaps and a periodic array of contact regions, where stick-slip contact occurs. The corresponding plane contact problem is reduced to a set of two singular integral equations. A solution to the contact problem is obtained for a certain shape of the grooves. The analysis of dependences of contact parameters of the solids on the applied load and gas pressure is carried out.
\end{abstract}

Keywords: stick-slip contact, periodically grooved surface, gas pressure

\section{Introduction}

The surface microtexturing, which consists in forming regularly (periodically) arranged grooves of the same shape on a surface of a solid, is one way of improving performance of joints. A regular surface texture may be generated by several methods (Etsion, 2004; Greco et al., 2009; Nakano et al., 2007; Schreck and Zum Gahr, 2005; Stepien, 2011): laser texturing, precise diamond turning, rolling, embossing, etching, vibrorolling, abrasive jet machining, micro electrical discharge machining, grinding. When periodically grooved surfaces are placed in contact, periodically arranged intercontact gaps occur at the interface. These gaps are usually filled with some substance (a natural substance (gas, liquid), a substance used for functional purposes (grease, coolant) or a biological fluid (synovia)). The effect of the interstitial medium filling the gaps of different nature on mechanical behavior of bodies was investigated by Kit et al. (2009), Machyshyn and Nagórko (2003), Martynyak and Slobodyan (2009), Evtushenko and Sulim (1981), Kaczyński and Monastyrskyy (2004), Monastyrskyy and Kaczyński (2007).

The contact between microtextured surfaces is usually realized not only under normal load, but also under shear load. This shear load may cause partial slip of surfaces, and the filler of the gaps may have some effect on propagation of the slip zones. However, the existing studies of stick-slip contact (Ciavarella, 1998a,b; Chang et al., 1984; Block and Keer, 2008; Hills et al., 2016; Goryacheva and Martynyak, 2014) do not consider this effect.

When contacting solids are subjected to heating, thermal deformations can also cause partial slip of surfaces. The thermally induced partial slip of a rigid flat-ended punch and an elastic half-space with different temperatures was investigated in (Pauk, 2007). The thermoelastic stickslip contact problem for two semi-infinite solids in the presence of a single thermoinsulated gap at the interface was studied in (Malanchuk et al., 2011). The effect of thermal conductivity of a medium filling the interface gaps on partial slip between a textured half-space and a flat half-space, which was caused by an imposed heat flow, was studied in (Chumak et al., 2014).

The goal of this research is to investigate partial slip between a half-space with a periodically grooved surface and a flat half-space, which is caused by the applied shear load, taking into 
account pressure of an ideal gas in the interface gaps. The stick-slip contact between a surface with a single groove and a flat surface in the presence of an ideal gas in the interface gap was previously studied in (Slobodyan et al., 2014). The model of partial slip between a periodically grooved surface and a flat one was presented in (Slobodyan et al., 2016). However, that model did not take into account the effect of the interstitial medium.

\section{Statement of the problem}

Consider the contact between two isotropic elastic half-spaces made of an identical material under plane strain conditions. The upper solid $D_{2}$ has a flat surface. The surface of the lower solid $D_{1}$ has an array of grooves of width $2 b$ spaced with the period $d(d>2 b)$. The shape of each groove is described by a smooth function $r(x)\left(r(x) \ll b, r^{\prime}(x) \ll 1, r( \pm b)=0, r^{\prime}( \pm b)=0\right)$. The solids are successively loaded by normal and shear loads. At the first stage of loading, the solids are pressed together by a monotonically increasing nominal pressure $p_{n}=P / d$ applied at infinity, where $P$ denotes the normal force per one period. The compressive load is assumed to vary quasistatically. Due to the regular surface texture of the lower solid, the interface consists of a periodic array of gaps and a periodic array of contacts. The width $2 a(a<b)$ and height $h(x)$ of the interface gaps are unknown and decrease monotonically with an increase of the nominal pressure. The gaps are filled with an equal amount of the ideal gas, whose pressure $P_{1}$ changes during the loading. The relation between the gas pressure $P_{l}$ and gas volume $V=l \int_{-a}^{a} h(x) d x$ is described by the ideal gas law

$$
P_{1} V=\frac{m_{1}}{\mu} R T
$$

where $m_{1}$ is mass of the gas, $\mu$ is molar mass of the gas, $T$ is gas temperature, $R$ is the ideal gas constant, $R=8.3145 \mathrm{JK}^{-1} \mathrm{~mol}^{-1}$ and $l=1 \mathrm{~m}$.

The shear displacements of the contacting surfaces due to the normal load are the same because of identity of the materials. The shear stresses do not, therefore, arise at the interface and the slip of the solids does not occur at the first loading phase. At the second loading phase, the normal load is held fixed and the bodies are subjected to the action of the nominal shear loading $s_{n}=S / d$ applied at infinity (Fig. 1). Here, $S$ denotes the shear force per one period. According to the Coulomb-Amontons law, the surfaces of the bodies are in stick until the contact shear stress $\tau_{x y}$ is less than the contact pressure $\left|\sigma_{y}\right|$ multiplied by the friction coefficient $f$ $\left(\tau_{x y}<f\left|\sigma_{y}\right|\right)$. The applied shear force leads to the frictional slip of the contacting surfaces in the regions $(-c+k d,-a+k d),(a+k d, c+k d)$, which, due to symmetry of the problem with respect to $y$-axis, are located symmetrically relative to the origin of each gap, $k=0, \pm 1, \pm 2, \ldots$. In the slip zones, $\tau_{x y}=f\left|\sigma_{y}\right|$. The direction of slip is indicated by arrows in Fig. 1.

Denote $I_{k}^{i}=[-i+k d, i+k d], J_{k}^{i}=[-d / 2+k d,-i+k d] \cup[i+k d, d / 2+k d], i=a, b, c$, $Y_{k}^{c, a}=[-c+k d,-a+k d] \cup[a+k d, c+k d]$, hereinafter $k=0, \pm 1, \pm 2, \ldots$

The boundary conditions are:

— at the gaps $\left(x \in I_{k}^{a}\right)$

$$
\begin{aligned}
& \sigma_{y}^{-}(x, 0)=\sigma_{y}^{+}(x, 0) \quad \sigma_{y}^{-}(x, 0)=-P_{1} \\
& \tau_{x y}^{-}(x, 0)=\tau_{x y}^{+}(x, 0) \quad \tau_{x y}^{-}(x, 0)=0
\end{aligned}
$$

— in the slip zones $\left(x \in Y_{k}^{c, a}\right)$

$$
\begin{aligned}
& \sigma_{y}^{-}(x, 0)=\sigma_{y}^{+}(x, 0) \quad \tau_{x y}^{-}(x, 0)=\tau_{x y}^{+}(x, 0) \\
& \tau_{x y}^{-}(x, 0)=-f \sigma_{y}^{-}(x, 0)
\end{aligned} \quad v^{-}(x, 0)-v^{+}(x, 0)=-r(x)
$$




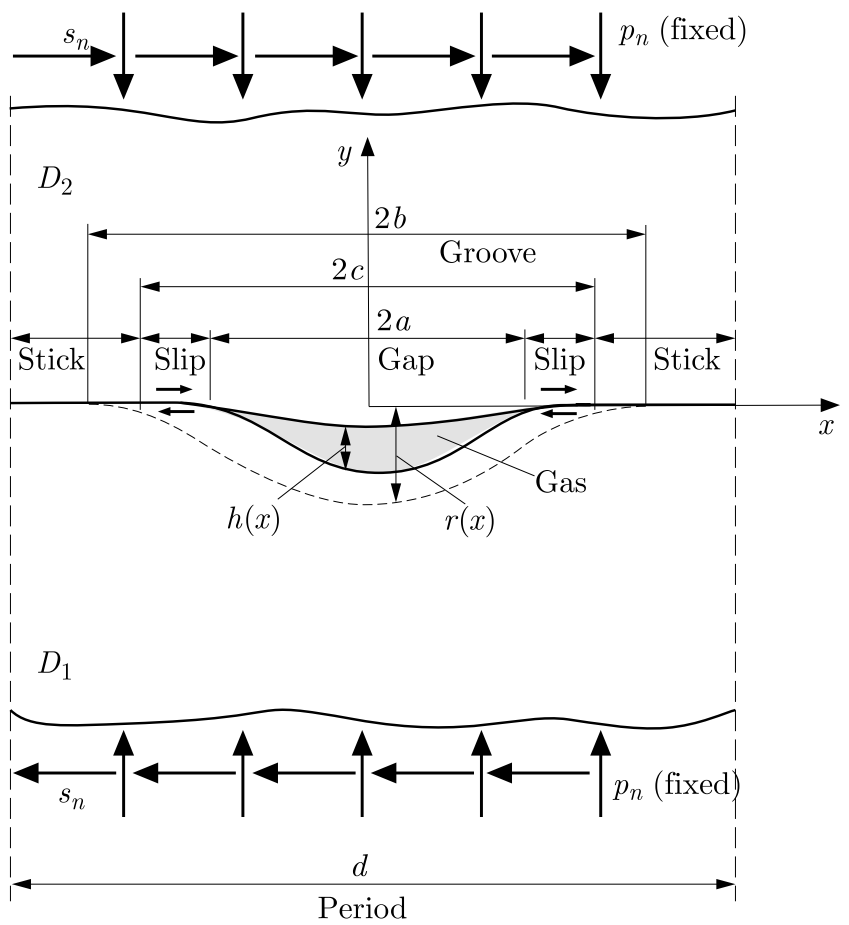

Fig. 1. Contact of the solids (within one period)

— in the stick zones $\left(x \in J_{k}^{c}\right)$

$$
\sigma_{y}^{-}(x, 0)=\sigma_{y}^{+}(x, 0) \quad \tau_{x y}^{-}(x, 0)=\tau_{x y}^{+}(x, 0) \quad v^{-}(x, 0)-v^{+}(x, 0)=-r(x)
$$

- at infinity

$$
\sigma_{y}(x, \pm \infty)=-p_{n} \quad \sigma_{x}(x, \pm \infty)=0 \quad \tau_{x y}(x, \pm \infty)=s_{n}
$$

Here, $r(x)=0$ when $x \in J_{k}^{b}, \sigma_{y}(x, y), \sigma_{x}(x, y), \tau_{x y}(x, y)$ are stress components, $u(x, y), v(x, y)$ are displacement components, superscripts + and - denote the boundary values of the functions on the $x$-axis in the upper and lower solid, respectively.

Note that because of identity of the contacting materials, the frictional slip does not influence the normal contact stress and width as well as and height of the gaps.

\section{Solution to the problem}

Let us represent the stresses and displacements in both solids throughout three functions: height of the grooves $r(x)$, height of the gaps $h(x)$, and relative tangential shift of the solids surfaces $U(x)=u^{-}(x, 0)-u^{+}(x, 0)$ (Slobodyan et al., 2014; Muskhelishvili, 1953)

$$
\begin{aligned}
& \sigma_{x}+\sigma_{y}=4 \operatorname{Re}\left[\Phi_{j}(z)\right]-p_{n} \\
& \sigma_{y}-\mathrm{i} \tau_{x y}=\Phi_{j}(z)-\Phi_{j}(\bar{z})+(z-\bar{z}) \overline{\Phi_{j}^{\prime}(z)}-p_{n}-\mathrm{i} s_{n} \\
& 2 G\left(u^{\prime}+\mathrm{i} v^{\prime}\right)=(3-4 \nu) \Phi_{j}(z)+\Phi_{j}(\bar{z})-(z-\bar{z}) \overline{\Phi_{j}^{\prime}(z)}+\nu p_{n}+\mathrm{i} s_{n} \\
& \Phi_{1}(z)=-\Phi_{2}(z)=\frac{(-1)^{j+1} G}{4 \pi(1-\nu)} \sum_{k=-\infty}^{\infty}\left(\mathrm{i} \int_{-c+k d}^{c+k d} \frac{U^{\prime}(t) d t}{t-z}+\int_{-a+k d}^{a+k d} \frac{h^{\prime}(t) d t}{t-z}+\int_{-b+k d}^{b+k d} \frac{r^{\prime}(t) d t}{t-z}\right) \\
& z \in D_{j} \quad j=1,2
\end{aligned}
$$

where $U(x)=0$ when $x \in J_{k}^{c}, z=x+\mathrm{i} y, \mathrm{i}=\sqrt{-1}, \nu$ is Poisson's ratio, $G$ is the shear modulus. 
Representations (3.1) have been constructed so that they satisfy boundary conditions $(2.3)_{1}$, (2.4) and (2.5). Taking into account the periodicity of the functions $U(x), h(x)$ and $r(x)$ (Schmueser and Comninou, 1979), the complex functions $\Phi_{1}(z), \Phi_{2}(z)$ can be rewritten as

$$
\begin{aligned}
& \Phi_{1}(z)=\frac{(-1)^{j+1} G}{4 d(1-\nu)}\left(\mathrm{i} \int_{-c}^{c} U^{\prime}(t) \cot \frac{\pi(t-z)}{d} d t+\int_{-a}^{a} h^{\prime}(t) \cot \frac{\pi(t-z)}{d} d t\right. \\
& \left.+\int_{-b}^{b} r^{\prime}(t) \cot \frac{\pi(t-z)}{d} d t\right) \\
& \Phi_{2}(z)=-\Phi_{1}(z) \quad z \in D_{j} \quad j=1,2
\end{aligned}
$$

The normal and shear stresses at the interface $y=0$ calculated from expressions (3.1), are

$$
\begin{aligned}
& N(x)=\sigma_{y}(x, 0)=\frac{G}{2 d(1-\nu)}\left(\int_{-b}^{b} r^{\prime}(t) \cot \frac{\pi(t-z)}{d} d t+\int_{-a}^{a} h^{\prime}(t) \cot \frac{\pi(t-z)}{d} d t\right)-p_{n} \\
& S(x)=\tau_{x y}(x, 0)=-\frac{G}{2 d(1-\nu)} \int_{-c}^{c} U^{\prime}(t) \cot \frac{\pi(t-z)}{d} d t+s_{n}
\end{aligned}
$$

In order to satisfy boundary condition $(2.2)_{1}$, we substitute $(3.3)_{1}$ into $(2.2)_{1}$, which leads to a singular integral equation

$$
\frac{1}{d} \int_{-a}^{a} h^{\prime}(t) \cot \frac{\pi(t-x)}{d} d t=-\frac{1}{d} \int_{-b}^{b} r^{\prime}(t) \cot \frac{\pi(t-x)}{d} d t+\frac{2(1-\nu)}{G}\left(p_{n}-P_{1}\right) \quad|x| \leqslant a
$$

From boundary conditions $(2.2)_{2}$ and $(2.3)_{2}$, and expression $(3.3)_{2}$, we obtain a singular integral equation for the function $U^{\prime}(x)$

$$
\begin{array}{ll}
\frac{1}{d} \int_{-c}^{c} U^{\prime}(t) \cot \frac{\pi(t-x)}{d} d t=\frac{2(1-\nu) s_{n}}{G} & \\
+ \begin{cases}0 & x \in I_{k}^{a} \\
& \left(\frac{1}{d} \int_{-a}^{a} h^{\prime}(t) \cot \frac{\pi(t-x)}{d} d t+\frac{1}{d} \int_{-b}^{b} r^{\prime}(t) \cot \frac{\pi(t-x)}{d} d t\right) \\
-\frac{2(1-\nu) f}{G}\left(p_{n}-P_{1}\right) & x \in Y_{k}^{c, a}\end{cases}
\end{array}
$$

As a result, we get a set of two singular integral equations with the Hilbert kernel for the functions $h^{\prime}(x)$ and $U^{\prime}(x)$.

It is obvious from Eq. (3.5) that the gas pressure $P_{1}$ influences the relative tangential shift $U(x)$ of the contacting surfaces.

The functions $h(x)$ and $U(x)$ satisfy the conditions

$$
\begin{array}{ll}
h( \pm a)=0 & h^{\prime}( \pm a)=0 \\
U( \pm c)=0 & U^{\prime}( \pm c)=0
\end{array}
$$

The first condition in $(3.6)_{1}$ means that the gaps between the solids vanish in the contact regions. The second condition in $(3.6)_{1}$ represents smooth closure of the gaps at $x= \pm a$ and 
ensures that the normal contact stress is bounded at $x= \pm a$. The first condition in $(3.6)_{2}$ follows from the continuity of shear displacements of the contacting surfaces. The second condition in $(3.6)_{2}$ ensures that the shear stress is bounded at the edges of the slip zones $x= \pm c$.

By changing variables $\xi=\tan (\pi x / d), \eta=\tan (\pi t / d), \alpha=\tan (\pi a / d), \beta=\tan (\pi b / d)$, $\gamma=\tan (\pi c / d)$, we reduce set (3.4), (3.5) to a set of singular integral equations with the Cauchy kernel

$$
\begin{aligned}
& \int_{-\alpha}^{\alpha} \frac{h^{\prime}(\eta) d \eta}{\eta-\xi}=-\int_{-\beta}^{\beta} \frac{r^{\prime}(\eta) d \eta}{\eta-\xi}+\frac{2 d(1-\nu)}{G\left(1+\xi^{2}\right)}\left(p_{n}-P_{1}\right) \quad|\xi| \leqslant \alpha \\
& \int_{-\gamma}^{\gamma} \frac{U^{\prime}(\eta) d \eta}{\eta-\xi}=\frac{2 d(1-\nu) s_{n}}{G\left(1+\xi^{2}\right)}+\left\{\begin{array}{cc}
0 & |\xi| \leqslant \alpha \\
f\left(\int_{-\alpha}^{\alpha} \frac{h^{\prime}(\eta) d \eta}{\eta-\xi}+\int_{-\beta}^{\beta} \frac{r^{\prime}(\eta) d \eta}{\eta-\xi}\right) & \\
-\frac{2 d(1-\nu) f}{G\left(1+\xi^{2}\right)}\left(p_{n}-P_{1}\right) & \alpha \leqslant|\xi| \leqslant \gamma
\end{array}\right.
\end{aligned}
$$

In the new variables, conditions (3.6) have the form

$$
\begin{array}{ll}
h( \pm \alpha)=0 & h^{\prime}( \pm \alpha)=0 \\
U( \pm \gamma)=0 & U^{\prime}( \pm \gamma)=0
\end{array}
$$

To solve set (3.7), the function $r(x)$, which describes the shape of the grooves, should be specified. We preset it as follows: $r(x)=-r_{0}\left(1-\tan ^{2}(\pi x / d) / \tan ^{2}(\pi b / d)\right)^{3 / 2}, x \in I_{k}^{b}$, where $r_{0}$ is the maximum depth of the grooves, and $0<r_{0} \ll b$. In the new variables, the shape of the grooves is $r(\xi)=-r_{0}\left(1-\xi^{2} / \beta^{2}\right)^{3 / 2},|\xi|<\beta$.

In accordance with the second condition in $(3.8)_{1}$, we find a solution to Eq. $(3.7)_{1}$ that is bounded at $\xi= \pm \alpha$. The bounded solution of a singular integral equation with the Cauchy kernel is possible only if the right-hand side of the equation satisfies the consistency condition (Muskhelishvili, 1953) for Eq. (3.7) 1

$$
\int_{-\alpha}^{\alpha}\left[\frac{-3 r_{0} \pi}{\beta}\left(\frac{1}{2}-\frac{\xi^{2}}{\beta^{2}}\right)+\frac{2 d(1-\nu)\left(p_{n}-P_{1}\right)}{G\left(1+\xi^{2}\right)}\right) \frac{d \xi}{\sqrt{\alpha^{2}-\xi^{2}}}=0
$$

Carrying out integrations, Eq. (3.9) reduces to an equation that relates the semi-width $\alpha$ of the gaps to the applied nominal pressure $p_{n}$

$$
\frac{3 r_{0} \pi}{\beta}\left(\frac{\alpha^{2}}{\beta^{2}}-1\right)+\frac{4 d(1-\nu)\left(p_{n}-P_{1}\right)}{G \sqrt{1+\alpha^{2}}}=0
$$

The bounded solution (Muskhelishvili, 1953) of Eq. (3.7) 1 is

$$
h^{\prime}(\xi)=\xi \sqrt{\alpha^{2}-\xi^{2}}\left(-\frac{3 r_{0}}{\beta^{3}}+\frac{2 d(1-\nu)\left(p_{n}-P_{1}\right)}{\pi G \sqrt{1+\alpha^{2}}\left(1+\xi^{2}\right)}\right) \quad|\xi| \leqslant \alpha
$$

Integration of Eq. (3.11) from $-\alpha$ to $\xi$ in view of the first condition in (3.8) 1 gives the gaps height $(|\xi| \leqslant \alpha)$

$$
h(\xi)=r_{0} \sqrt{\left(\frac{\alpha^{2}}{\beta^{2}}-\frac{\xi^{2}}{\beta^{2}}\right)^{3}}+\frac{d(1-\nu)\left(p_{n}-P_{1}\right)}{\pi G}\left(\frac{2 \sqrt{\alpha^{2}-\xi^{2}}}{\sqrt{1+\alpha^{2}}}-\ln \left|\frac{\sqrt{1+\alpha^{2}}+\sqrt{\alpha^{2}-\xi^{2}}}{\sqrt{1+\alpha^{2}}-\sqrt{\alpha^{2}-\xi^{2}}}\right|\right)
$$


Substituting Eq. (3.12) into the formula for gas volume and carrying out integration, ideal gas law (2.1) can be rewritten as

$$
P_{1}\left[\frac{\pi r_{0}}{\beta^{3}}\left(\sqrt{\left(1+\alpha^{2}\right)^{3}}-1-\frac{3}{2} \alpha^{2}\right)+\frac{d(1-\nu)\left(p_{n}-P_{1}\right)}{G}\left(2-\frac{2}{\sqrt{1+\alpha^{2}}}-\ln \left(1+\alpha^{2}\right)\right)\right]=\frac{m_{1}}{\mu} R T
$$

Note that the function $h(\xi)$ includes two unknown parameters: the parameter $\alpha$ and gas pressure $P_{1}$. For determination of these parameters, we use (3.10) and (3.13). Since Eqs. (3.10) and (3.13) are nonlinear with respect to $\alpha$ and the difference $p_{n}-P_{1}$ appears in both equations, the following technique is proposed for solving set (3.10), (3.13):

i) the external load $p_{n}$ is assumed to be unknown, while the gaps width $\alpha$ is set from the range $0<\alpha \leqslant \beta$;

ii) the difference between the external load $p_{n}$ and the gas pressure $P_{1}$ is then determined from Eq. (3.10) as

$$
p_{n}-P_{1}=\frac{3 \pi G r_{0} \sqrt{1+\alpha^{2}}}{4(1-\nu) \beta^{3} d}\left(\beta^{2}-\alpha^{2}\right)
$$

the gas pressure $P_{1}$ is then obtained from Eq. (3.13) after substituting (3.14) for $p_{n}-P_{1}$

$$
P_{1}=\frac{\beta^{3} m_{1} R T}{2 \pi \mu r_{0}}\left(\left(\sqrt{1+\alpha^{2}}-1\right)\left(3 \beta^{2}+2\right)-\frac{\sqrt{\alpha^{2}+1}}{2}\left[2 \alpha^{2}+3\left(\beta^{2}-\alpha^{2}\right) \ln \left(1+\alpha^{2}\right)\right]\right)^{-1}
$$

iii) once the gas pressure $P_{1}$ for a given value of $\alpha$ is known, the external load $p_{n}$ is calculated from Eq. (3.10) as

$$
p_{n}=P_{1}+\frac{3 \pi G r_{0} \sqrt{1+\alpha^{2}}}{4(1-\nu) \beta^{3} d}\left(\beta^{2}-\alpha^{2}\right)
$$

Substituting Eq. (3.11) into Eq. (3.3) 1 , we find the normal contact stress:

- for $\alpha \leqslant|\xi| \leqslant \beta$

$$
N(\xi)=\frac{3 \pi G r_{0}\left(1+\xi^{2}\right)}{4 d(1-\nu) \beta^{3}}\left(\frac{\sqrt{1+\alpha^{2}}+|\xi| \sqrt{\xi^{2}-\alpha^{2}}}{1+\xi^{2}}\left(\beta^{2}-\alpha^{2}\right)-2|\xi| \sqrt{\xi^{2}-\alpha^{2}}\right)-p_{n}
$$

- for $|\xi| \geqslant \beta$

$$
N(\xi)=\frac{3 \pi G r_{0}\left(1+\xi^{2}\right)}{4 d(1-\nu) \beta^{3}}\left[\frac{\sqrt{1+\alpha^{2}}+|\xi| \sqrt{\xi^{2}-\alpha^{2}}}{1+\xi^{2}}\left(\beta^{2}-\alpha^{2}\right)+2|\xi|\left(\sqrt{\xi^{2}-\beta^{2}}-\sqrt{\xi^{2}-\alpha^{2}}\right)\right]-p_{n}
$$

By taking Eqs. (3.15) and (3.16) into account, Eq. (3.7) 2 appears as follows

$$
\int_{-\gamma}^{\gamma} \frac{U^{\prime}(\eta) d \eta}{\eta-\xi}=\frac{2 d(1-\nu) s_{n}}{G\left(1+\xi^{2}\right)}+L(\xi) \quad|\xi| \leqslant \gamma
$$


where

$$
\begin{aligned}
L(\xi) \equiv \begin{cases}0 & |\xi| \leqslant \alpha \\
F(\xi) & \alpha \leqslant|\xi| \leqslant \gamma\end{cases} \\
F(\xi)= \begin{cases}\frac{3 f r_{0} \pi}{2 \beta^{3}}\left(\frac{\sqrt{1+\alpha^{2}}+|\xi| \sqrt{\xi^{2}-\alpha^{2}}}{1+\xi^{2}}\left(\beta^{2}-\alpha^{2}\right)-2|\xi| \sqrt{\xi^{2}-\alpha^{2}}\right)-f p_{n} \quad \xi \leqslant \beta \\
\frac{3 f r_{0} \pi}{2 \beta^{3}}\left(\frac{\sqrt{1+\alpha^{2}}+|\xi| \sqrt{\xi^{2}-\alpha^{2}}}{1+\xi^{2}}\left(\beta^{2}-\alpha^{2}\right)\right. \\
\left.+2|\xi|\left(\sqrt{\xi^{2}-\beta^{2}}-\sqrt{\xi^{2}-\alpha^{2}}\right)\right)-f p_{n} & \xi>\beta\end{cases}
\end{aligned}
$$

Since conditions (3.8) $)_{2}$ must be met, we find a solution to Eq. (3.17) that is bounded at $\xi= \pm \gamma$. The solution to singular integral equation (3.17) is (Muskhelishvili, 1953)

$$
U^{\prime}(\xi)=\frac{2 d(1-\nu) s_{n} \xi \sqrt{\gamma^{2}-\xi^{2}}}{\pi G \sqrt{1+\gamma^{2}}\left(1+\xi^{2}\right)}-\frac{\sqrt{\gamma^{2}-\xi^{2}}}{\pi^{2}} \int_{-\gamma}^{\gamma} \frac{L(\eta) d \eta}{\sqrt{\gamma^{2}-\eta^{2}}(\eta-\xi)} \quad|\xi| \leqslant \gamma
$$

Using a piecewise constant approximation of the function $L(\xi)$ for evaluation of the integral in the right-hand side of Eq. (3.18), we obtain

$$
U^{\prime}(\xi)=\frac{2 d(1-\nu) s_{n} \xi \sqrt{\gamma^{2}-\xi^{2}}}{\pi G\left(1+\xi^{2}\right) \sqrt{1+\gamma^{2}}}+\frac{1}{2 \pi^{2}} \sum_{j=0}^{m-1} L_{j}\left(\Gamma\left(\gamma, \xi, \zeta_{j+1}\right)-\Gamma\left(\gamma, \xi, \zeta_{j}\right)\right) \quad|\xi| \leqslant \gamma
$$

where $\zeta_{j}=-\gamma+2 j \gamma / m, j=1,2, \ldots, m$ are nodes of the approximation; $L_{j}$ are the nodal values of the function $L(\xi)$, that is $L_{j}=L\left(\zeta_{j}\right), j=1,2, \ldots, m$; and

$$
\Gamma(\gamma, \xi, \zeta)=\ln \frac{\gamma^{2}-\xi \zeta+\sqrt{\left(\gamma^{2}-\xi^{2}\right)\left(\gamma^{2}-\zeta^{2}\right)}}{\gamma^{2}-\xi \zeta-\sqrt{\left(\gamma^{2}-\xi^{2}\right)\left(\gamma^{2}-\zeta^{2}\right)}}
$$

Integration of Eq. (3.19) from $-\gamma$ to $\xi$ in view of the first condition in Eq. (3.8) $)_{2}$ gives the relative tangential shift

$$
\begin{aligned}
& U(\xi)=\frac{d(1-\nu) s_{n}}{\pi G}\left(\ln \left|\frac{\sqrt{1+\gamma^{2}}-\sqrt{\gamma^{2}-\xi^{2}}}{\sqrt{1+\gamma^{2}}+\sqrt{\gamma^{2}-\xi^{2}}}\right|+\frac{2 \sqrt{\gamma^{2}-\xi^{2}}}{\sqrt{1+\gamma^{2}}}\right) \\
& +\frac{1}{\pi^{2}} \sum_{j=0}^{m-1} L_{j}\left\{\left(\xi-\zeta_{j+1}\right) \Gamma\left(\gamma, \xi, \zeta_{j+1}\right)-\left(\xi-\zeta_{j}\right) \Gamma\left(\gamma, \xi, \zeta_{j}\right)\right. \\
& \left.+2\left(\sqrt{\gamma^{2}-\zeta_{j+1}^{2}}-\sqrt{\gamma^{2}-\zeta_{j}^{2}}\right)\left[\arcsin \left(\frac{\xi}{\gamma}\right)+\frac{\pi}{2}\right]\right\} \quad|\xi| \leqslant \gamma
\end{aligned}
$$

By setting $\xi=\gamma$ in Eq. (3.20) and in view of the first condition in Eq. (3.8) $)_{2}$, we obtain an equation for width $\gamma$ of the slip zones

$$
\frac{2 d(1-\nu) s_{n}}{G \sqrt{1+\gamma^{2}}}+\frac{1}{\pi^{2}} \sum_{j=0}^{m-1} L_{j}\left(\sqrt{\gamma^{2}-\zeta_{j+1}^{2}}-\sqrt{\gamma^{2}-\zeta_{j}^{2}}\right)=0
$$

This equation is solved numerically. Substituting Eq. (3.19) into Eq. (3.3) $)_{2}$ and performing some integrations, we find shear contact stresses in the stick zones $(|\xi| \geqslant \gamma)$

$$
\begin{aligned}
& S(\xi)=\frac{s_{n}}{\sqrt{1+\gamma^{2}}}\left(\xi^{2}+1-|\xi| \sqrt{\xi^{2}-\gamma^{2}}\right)+\frac{G}{2 d(1-\nu) \pi}\left(\xi^{2}+1\right) \\
& \cdot \sum_{j=0}^{m-1} L_{j}\left[\arcsin \left(\frac{\zeta_{j+1}}{\gamma}\right)-\arcsin \left(\frac{\zeta_{j}}{\gamma}\right)-\arcsin \left(\frac{\xi \zeta_{j+1}-\gamma^{2}}{\gamma\left(\xi-\zeta_{j+1}\right)}\right)+\arcsin \left(\frac{\xi \zeta_{j}-\gamma^{2}}{\gamma\left(\xi-\zeta_{j}\right)}\right)\right]
\end{aligned}
$$


As $d \rightarrow \infty$, we obtain results for the single groove (Slobodyan et al., 2014). By putting in Eqs. (3.10)-(3.12) and (3.15)-(3.22) $m_{1}=0$, we obtain results for the frictional contact interaction between two solids, one of which having a regular surface texture in form of periodically arranged grooves, in the case when the gaps do not contain a filler (Slobodyan et al., 2016).

\section{Numerical results and discussion}

The obtained results are illustrated in Figs. 2-6, where the dimensionless parameters $\bar{x}=x / d$, $\bar{a}=a / d, \bar{b}=b / d, \bar{c}=c / d, \bar{r}=r / d, \bar{h}=h / d, \bar{U}=U / d, \bar{\sigma}_{y}=4 \sigma_{y}(1-\nu) G^{-1}, \bar{\tau}_{x y}=4 \tau_{x y}(1-$ $\nu) G^{-1}, \bar{p}_{n}=4 p_{n}(1-\nu) G^{-1}, \bar{s}_{n}=4 s_{n}(1-\nu) G^{-1}, \bar{P}_{1}=4 P_{1}(1-\nu) G^{-1}, \bar{m}_{1}=m_{1} R T \mu^{-1} d^{-1}$ are introduced. The maximum depth of the grooves $\bar{r}_{0}$ is taken to be $10^{-3}$, the half-width of the grooves $\bar{b}=0.3$, and the friction coefficient $f=0.1$.

The nonlinear dependence of the half-width $\bar{a}$ of the gaps on the applied pressure $\bar{p}_{n}$ is given in Fig. 2. The half-width $\bar{a}$ of the gaps decreases with an increase in the applied pressure $\bar{p}_{n}$.

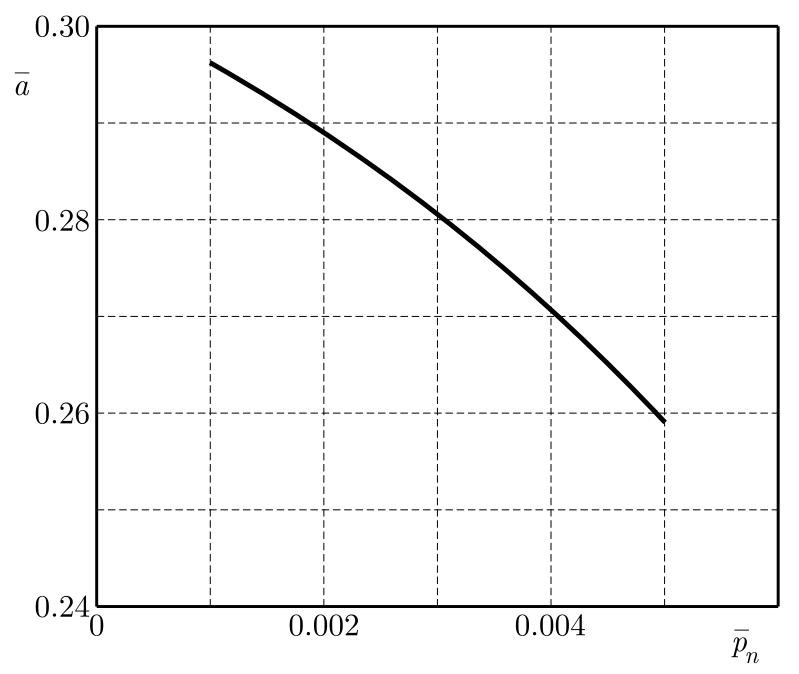

Fig. 2. Dependence of the half-width $\bar{a}$ of the gaps on the applied pressure $\bar{p}_{n}$

Figure 3a shows height of the gaps for different values of the applied pressure $\bar{p}_{n}$. The height $\bar{h}$ of the gaps decreases with the increasing nominal pressure and has its maximum value in the center of the gap. The height $\bar{h}$ of the gaps for different values of the gas mass $\bar{m}_{1}$ is given in Fig. $3 \mathrm{~b}$, where $\bar{p}_{n}=0.003$. The curve for $\bar{m}_{1}=0$ corresponds to the case of gaps without a filler. As seen in the figure, $\bar{h}$ is the largest in the case of filled gaps and increases with increasing $\bar{m}_{1}$.

Figure 4a shows the dependence of the half-width $\bar{c}$ of the slip zones on the shear load $\bar{s}_{n}$ for different values of the applied pressure $\bar{p}_{n}$. If the edge of the slip zone is located within the groove $(\bar{a}<\bar{c}<\bar{b})$, then this dependence is nonlinear. If the edge of the slip zone exceeds the bounds of the groove $(\bar{c}>\bar{b})$, then this dependence is almost linear. The width $\bar{c}$ of the slip zones monotonically increases with the increasing shear load $\bar{s}_{n}$.

The rate of the increase of $\bar{c}$ with a change of $\bar{s}_{n}$ is greater for smaller values of $\bar{p}_{n}$. The curves $\bar{c}=\bar{c}\left(\bar{s}_{n}\right)$ have specific kinks at the points $\bar{c}=\bar{b}=0.3$ (the right/left end of the right/left slip zone reaches the right/left end of the groove). If $\bar{c}>\bar{b}$, then the slope of curves $\bar{c}=\bar{c}\left(\bar{s}_{n}\right)$ increases significantly. This effect can be explained by the fact that the normal contact stress has its maximum value at the edges of the grooves and rapidly decreases in the region beyond the grooves (see Fig. 6a). The dependence of the half-width $\bar{c}$ of the slip zones on the gas mass $\bar{m}_{1}$ is given in Fig. $4 \mathrm{~b}$, where $\bar{p}_{n}=0.003$. As seen in the figure, for a fixed $\bar{s}_{n}, \bar{c}$ is the smallest in the case of gaps without a filler (the curve that corresponds to $\bar{m}_{1}=0$ ) and increases with increasing $\bar{m}_{1}$ (curves that correspond to $\bar{m}_{1}=8 \cdot 10^{-7}, \bar{m}_{1}=9 \cdot 10^{-7}, \bar{m}_{1}=10^{-6}$ ). 
(a)

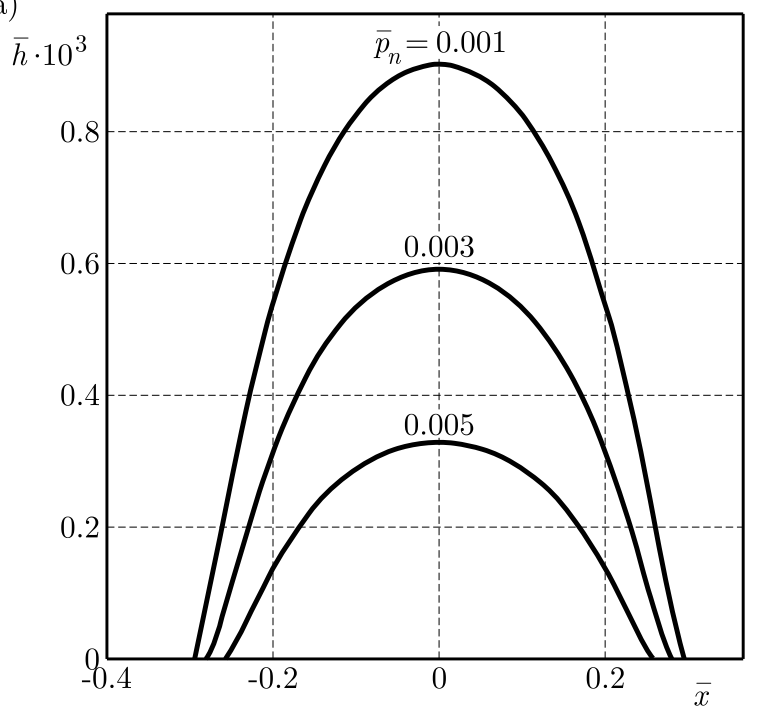

(b)

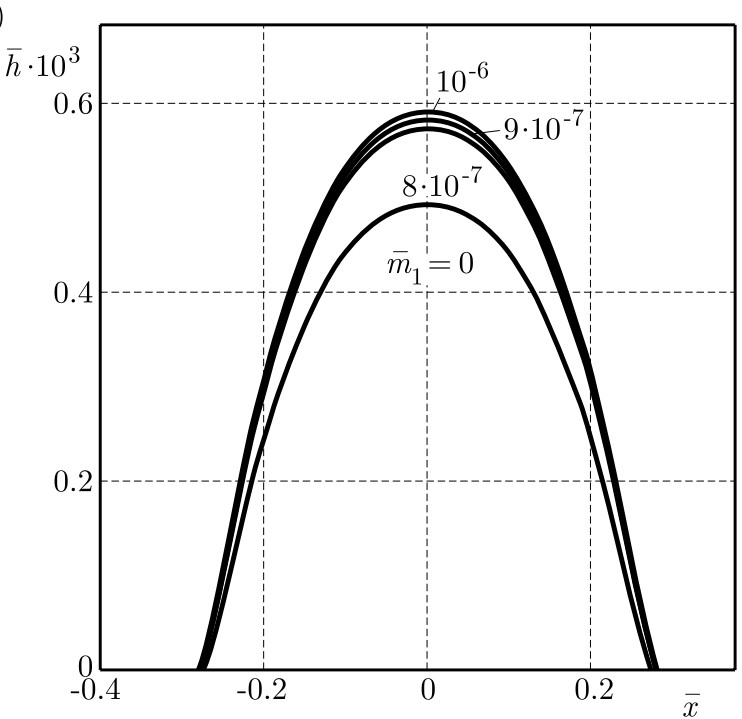

Fig. 3. The height $\bar{h}$ of the gaps

(a)

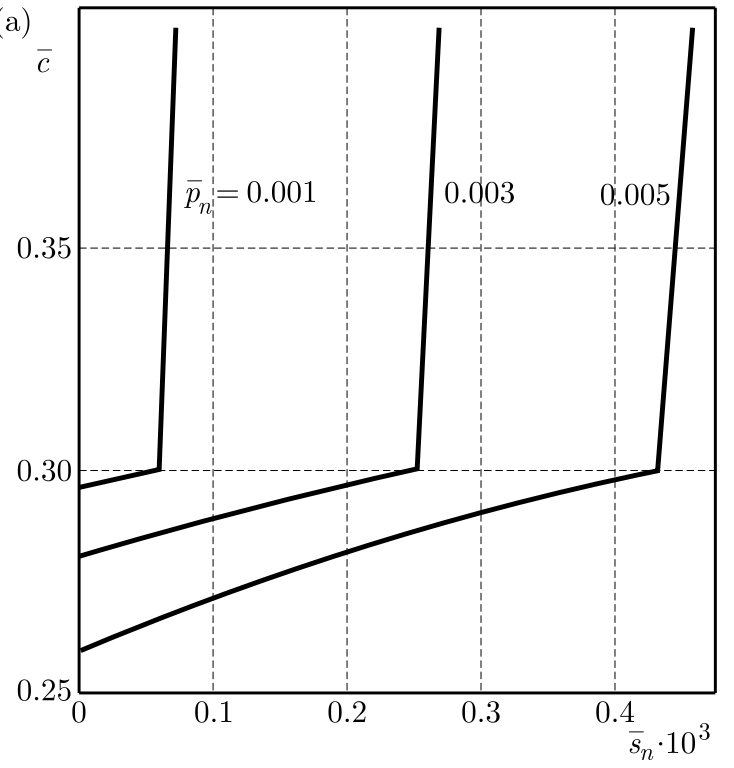

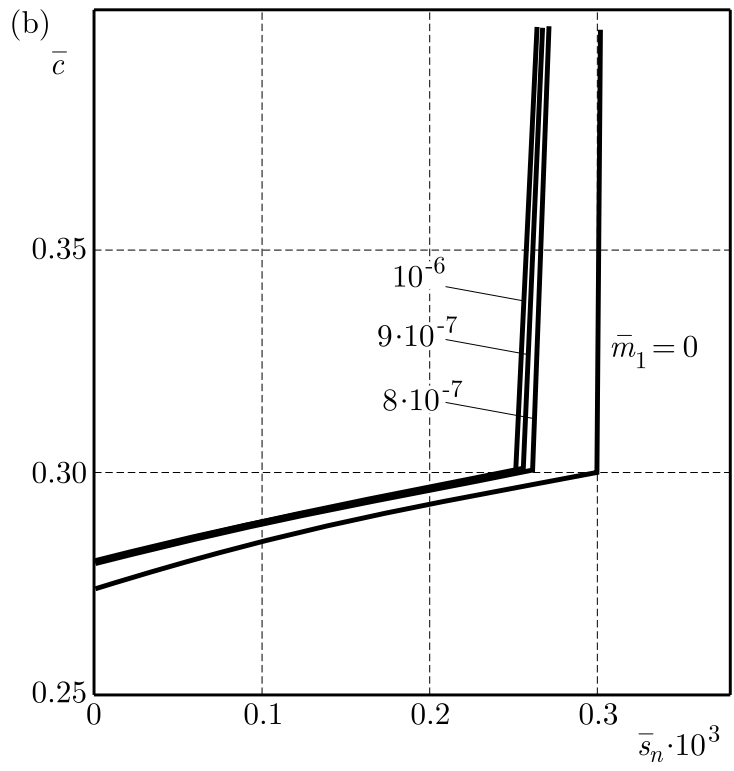

Fig. 4. Dependence of the half-width $\bar{c}$ of the slip zones on the shear load $\bar{s}_{n}$

Figure 5a shows the relative tangential shift $\bar{U}$ of the surfaces within one period for different values of the applied pressure $\bar{p}_{n}$. The relative tangential shift $\bar{U}$ of the surfaces increases with the increasing applied pressure $\bar{p}_{n}$ and has its maximum value in the center of the grooves. At the gaps, the relative tangential shift $\bar{U}$ is larger than in the slip zones. The relative tangential shift $\bar{U}$ of the surfaces within one period for four values of the gas mass $\bar{m}_{1}\left(0 ; 8 \cdot 10^{-7} ; 9 \cdot 10^{-7} ; 10^{-6}\right)$ is given in Fig. 5b, where $\bar{p}_{n}=0.001$. The relative tangential shift $\bar{U}$ is smallest in the case of gaps without the filler (the curve that corresponds to $\bar{m}_{1}=0$ ) and increases with increasing $\bar{m}_{1}$ (curves that correspond to $\bar{m}_{1}=8 \cdot 10^{-7}, \bar{m}_{1}=9 \cdot 10^{-7}, \bar{m}_{1}=10^{-6}$ ).

Figure 6a shows the distribution of the contact normal stress $\bar{\sigma}_{y}$ for different values of the nominal pressure $\bar{p}_{n}$. The magnitude of the contact normal stress $\left|\bar{\sigma}_{y}\right|$ increases with the increasing nominal pressure $\bar{p}_{n}$ and has its maximum value at the edges of the grooves $(\bar{x}=\bar{b}=$ $\pm 0.3)$. As required by boundary condition $(2.2)_{1}, \sigma_{y}^{ \pm}=-P_{1}$ at the surfaces of the gaps. Beyond the grooves, the contact normal stress monotonically decreases. 
(a)

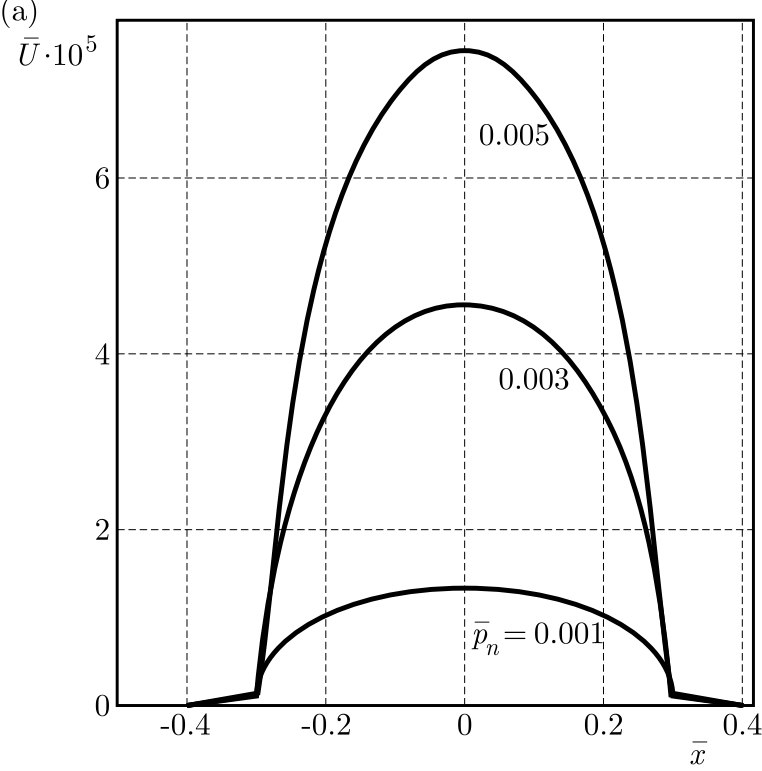

(b)

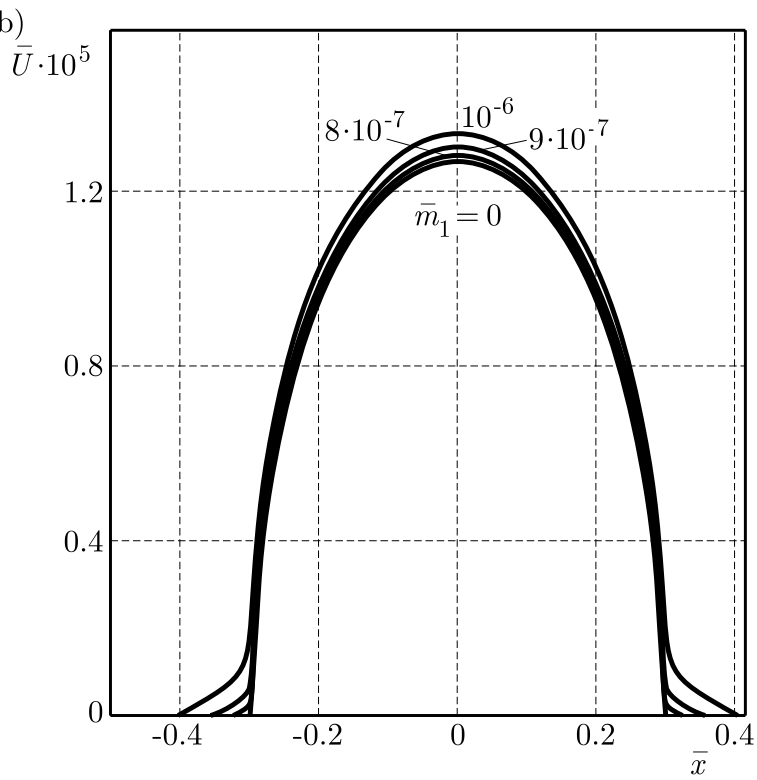

Fig. 5. The relative tangential shift $\bar{U}$ of the surfaces

(a)

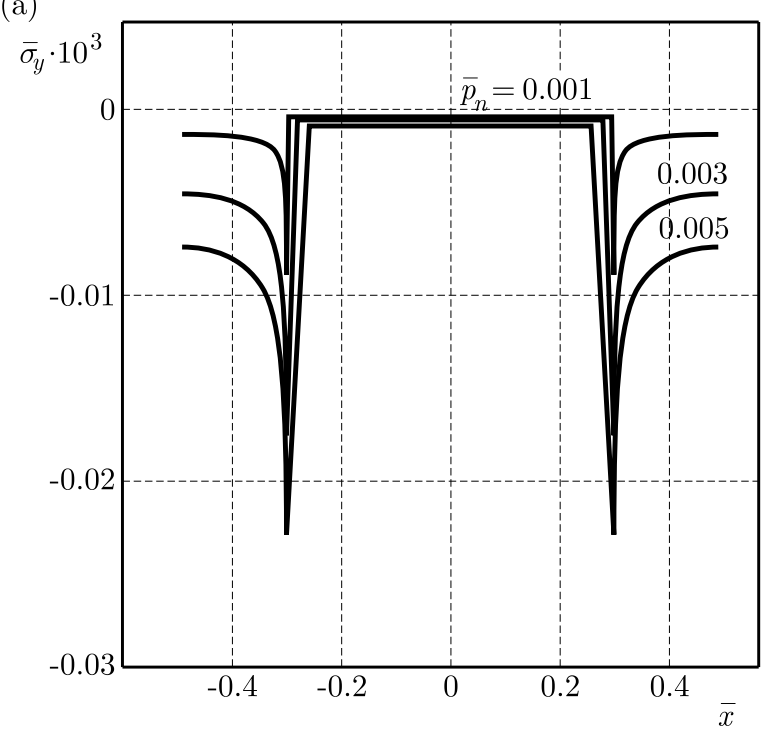

(b)

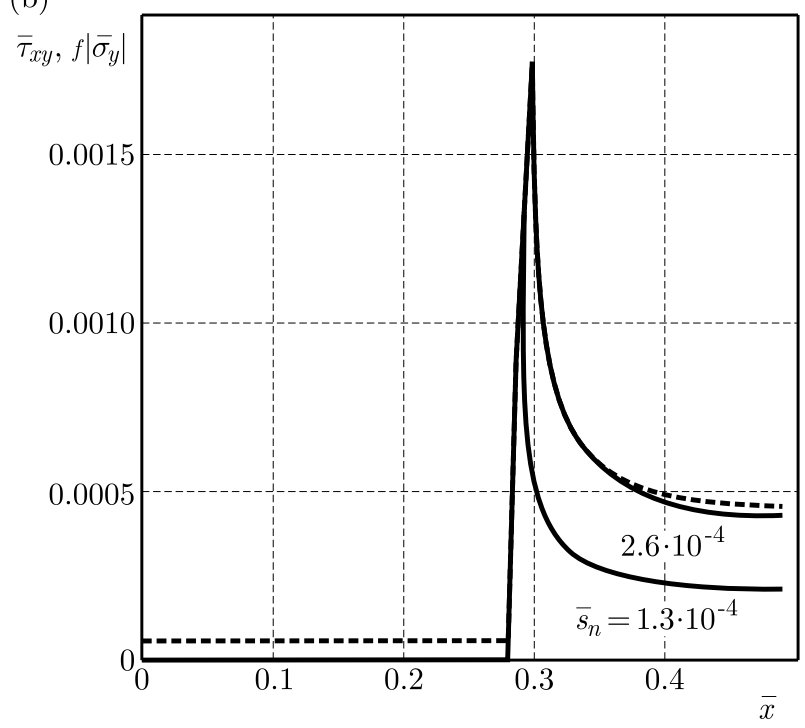

Fig. 6. Distribution of the stresses

The distributions of the shear contact stress $\bar{\tau}_{x y}$ (solid curves) and the normal contact stress $f\left|\bar{\sigma}_{y}\right|$ multiplied by the friction coefficient (dashed curve) are given in Fig. 6b. The solid curves are plotted for two values of the shear load $\bar{s}_{n}$. For $\bar{s}_{n}=1.3 \cdot 10^{-4} ; 2.6 \cdot 10^{-4}$, the corresponding values of $\bar{c}$ are $0.28 ; 0.35$. The shear contact stress $\bar{\tau}_{x y}$ increases with the increasing shear load $\bar{s}_{n}$. The stress $\bar{\tau}_{x y}$ has the maximum value at $\bar{x}= \pm \bar{c}$ (the ends of the slip zones) if the applied shear load $\bar{s}_{n}$ is such that slip occurs within the grooves $(\bar{c}<\bar{b})$ (the curve that corresponds to $\bar{s}_{n}=1.3 \cdot 10^{-4}$ ), and at $\bar{x}= \pm \bar{b}$ (the ends of the grooves) if the applied shear load $\bar{s}_{n}$ is such that slip extends outside the grooves $(\bar{c} \geqslant \bar{b}$ ) (the curve that corresponds to $\left.\bar{s}_{n}=2.6 \cdot 10^{-4}\right)$. At the surfaces of the gaps, $\bar{\tau}_{x y}=0$ and $f\left|\bar{\sigma}_{y}\right|=f P_{1}$. As required by boundary condition $(2.3)_{2}$, the solid curves coincide with the dashed curve in the slip zones. Outside the slip zones, $\bar{\tau}_{x y}$ is less than $f\left|\bar{\sigma}_{y}\right|$ (the solid curves lie below the dashed curve). 


\section{Conclusions}

The contact problem for two half-spaces of the same material under sequential application of the nominal pressure and nominal shear stress has been considered. One of the contacting surfaces is textured in form of periodically arranged grooves, and the other is flat. Partial slip of the surfaces of the solids is induced by shear load, and the first points to slip are the ends of each gap. The contact problem has been solved for the case when the interface gaps are filled with the ideal gas. Analysis of contact parameters of the contact pair on the applied load has been carried out. It has been shown that the half-width and height of the gaps decrease when the nominal pressure increases. The height of the gaps is the largest in the case of filled gaps and increases with the increasing mass of the gas, and has the maximum value in the center of the gaps. The normal contact stress increases with the increasing nominal pressure and has the maximum value at the edges of grooves. The width of the slip zones, relative tangential shift of the surfaces and shear contact stress increase with the increasing shear load. For a fixed load, the relative tangential shift and width of the slip zones is the smallest in the case of gaps without the filler and increases with the increasing gas mass. The shear contact stress has the maximum value at the ends of the slip zones if the applied shear load is such that slip occurs within the grooves and at the ends of the grooves if the applied shear load is such that slip extends outside the grooves.

\section{References}

1. Block J.M., Keer L.M., 2008, Periodic contact problems in plane elasticity, Journal of Mechanics of Materials and Structures, 3, 7, 1207-1237

2. Chang F.-K., Comninou M., Sheppard S., Barber J.R., 1984, The subsurface crack under conditions of slip and stick caused by a surface normal force, ASME Journal of Applied Mechanics, 51, 311-316

3. Chumak K., Malanchuk N., Martynyak R., 2014, Partial slip contact problem for solids with regular surface texture assuming thermal insulation or thermal permeability of interface gaps, International Journal of Mechanical Sciences, 84, 138-146

4. Ciavarella M., 1998a, The generalized Cattaneo partial slip plane contact problem. I - Theory, International Journal of Solids and Structures, 35, 18, 2349-2362

5. Ciavarella M., 1998b, The generalized Cattaneo partial slip plane contact problem. II - Examples, International Journal of Solids and Structures, 35, 18, 2363-2378

6. Etsion I., 2004, Improving tribological performance of mechanical components by laser surface texturing, Tribology Letters, 17, 4, 733-737

7. Evtushenko A.A., Sulim G.T., 1981, Stress concentration near a cavity filled with a liquid, Materials Science, 16, 6, 546-549

8. Goryacheva I.G., Martynyak R.M., 2014, Contact problems for textured surfaces involving frictional effects, Proceedings of the Institution of Mechanical Engineers, Part J: Journal of Engineering Tribology, 228, 7, 707-716

9. Greco A., Raphaelson S., Ehmann K., Wang Q.J., Lin C., 2009, Surface texturing of tribological interfaces using the vibromechanical texturing method, ASME Journal of Manufacturing Science and Engineering, 131, 6, 061005-1-061005-8

10. Hills D.A., Fleury R.M.N., Dini D., 2016, Partial slip incomplete contacts under constant normal load and subject to periodic loading, International Journal of Mechanical Sciences, 108109, $115-121$ 
11. Kaczyński A., Monastyrskyy B., 2004, On the problem of some interface defect filled with a compressible fluid in a periodic stratified medium, Journal of Theoretical and Applied Mechanics, 42, $1,41-57$

12. Kit G.S., Martynyak R.M., Machishin I.M., 2009, The effect of a fluid in the contact gap on the stress state of conjugate bodies, International Applied Mechanics, 39, 3, 292-299

13. Machyshyn I., NAgóRKo W., 2003, Interaction between a stratified elastic half-space and an irregular base allowing for the intercontact gas, Journal of Theoretical and Applied Mechanics, 41, 2, 271-288

14. Malanchuk N., Martynyak R., Monastyrskyy B., 2011, Thermally induced local slip of contacting solids in vicinity of surface groove, International Journal of Solids and Structures, 48, $11 / 12,1791-1797$

15. Martynyak R.M., Slobodyan B.S., 2009, Contact of elastic half spaces in the presence of an elliptic gap filled with liquid, Materials Science, 45, 1, 62-66

16. Monastyrskyy B., Kaczyński A., 2007, The elasticity problem for a stratified semi-infinite medium containing a penny-shaped crack filled with a gas, Acta Mechanica et Automatica, 1, 1, $63-66$

17. Muskhelishvili N.I., 1953, Singular Integral Equations: Boundary Problems of Function Theory and Their Application to Mathematical Physics, P. Noordhoff, N.V. Groningen

18. Nakano M., Korenaga A., Korenaga A., Miyake K., Murakami T., Ando Y., Usami H., SAsAKI S., 2007, Applying micro-texture to cast iron surfaces to reduce the friction coefficient under lubricated conditions, Tribology Letters, 28, 2, 131-137

19. PAUK V., 2007, Plane contact of hot flat-ended punch and thermoelastic half-space involving finite friction, ASME Journal of Applied Mechanics, 74, 6, 1172-1177

20. Schmueser D., Comninou M., 1979, The periodic array of interface cracks and their interaction, International Journal of Solids and Structures, 15, 12, 927-934

21. Schreck S., Zum Gahr K.-H., 2005, Laser-assisted structuring of ceramic and steel surfaces for improving tribological properties, Applied Surface Science, 247, 1-4, 616-622

22. Slobodyan B.S., Lyashenko B.A., Malanchuk N.I., Marchuk V.E., Martynyak R.M., 2016, Modeling of contact interaction of periodically textured bodies with regard for frictional slip, Journal of Mathematical Sciences, 215, 1, 110-120

23. Slobodyan B.S., Malanchuk N.I., Martynyak R.M., Lyashenko B.A., Marchuk V.E., 2014, Local sliding of elastic bodies in the presence of gas in the intercontact gap, Materials Science, 50, 2, 261-268

24. Stepien P., 2011, Deterministic and stochastic components of regular surface texture generated by a special grinding process, Wear, 271, 3/4, 514-518 